\title{
Billroth I Procedure
}

National Cancer Institute

\section{Source}

National Cancer Institute. Billroth I Procedure. NCI Thesaurus. Code C51581.

Surgical removal of the lower part of the stomach with end-to-end anastomosis of the remaining segment of the stomach with the duodenum. 